\title{
Inverse Kinematics of a Postprocessor for Five-Axis Machine Tools Yuan-Lung Lai ${ }^{1, a^{*}}$, Chien-Chih Liao ${ }^{12, b}$, Hsiao-Ying Chan ${ }^{1, \mathrm{c}}$ and Ching-Kuei $\mathrm{Su}^{3, \mathrm{~d}}$
}

${ }^{1}$ Department of Industrial Education and Technology, National Changhua University of Education, Changhua, Taiwan, R.O.C.

${ }^{2}$ Intelligent Machine Tool Technology Center, Industrial Technology Research Institute, Taichung, Taiwan, R.O.C.

${ }^{3}$ Research and Development Department, Wishing Technology Co., Ltd., Taichung, Taiwan, R.O.C.

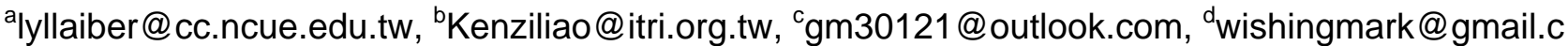
om

Keywords: Five-axis, Modified D-H notation, Inverse kinematic, Postprocessor

\begin{abstract}
This paper presents a modular method to construct the postprocessor system for a novel hybrid parallel-serial five-axis machine tool. A hybrid parallel-serial mathematic model was introduced to analyze the structural configuration. The configuration decomposition of machine tools is employed to create the kernel of postprocessor. The proposed modified D-H notation and screw theory are used for coordinate conversion procedure and then an algorithm for developing the inverse kinematics of five-axis machines was carried out. CL path is converted to produce NC Code and been loaded into the controller for machining. This proposed algorithm can be easily adapted to convert between CL path and NC code and be implemented on computerized CAD/CAM systems. Examples with side-milling tools are demonstrated and real cutting parts are implemented for verifying the algorithm.
\end{abstract}

\section{Introduction}

Generally mechanical kinematics is divided into two categories, direct kinematics and inverse kinematics. Inverse kinematics the location of the end-effector is given and the target is to find the joint variables. In the direct kinematics the joint variables are given and the problem is to calculate the position of the end-effector[1-4].

Owing to the simultaneous interpolation movement both with the linear and rotary axes on a fiveaxis machine, the derivation of the five-axis feeding path is more complex than that of the threeaxis feeding path. Therefore a postprocessor must be utilized to transfer the cutter location (CL) data from the CAM system into the machine control data. Although the advanced controllers can accept the CL data to machine the working piece in real-time without the need of postprocessor [5], they are relatively expensive and only used in some specific works. The postprocessor may be positioned within the flow chart shown in Fig. 1, which illustrates the concept that the postprocessor is not definitely a part of the CAM system, but can also be a stand-alone system.

Hybrid Five-Axis Machine Tool. Simplified the structure, a machine tool may be regard as a set of links joined in a set of connectors. Standard definition of five-axis machine coordinate frame consists of the $\mathrm{X}, \mathrm{Y}$ and $\mathrm{Z}$ axes in right-hand rectangular coordinates. The rotary axes are defined as $\mathrm{A}$ axis, $\mathrm{B}$ axis and $\mathrm{C}$ axis which rotate about the $\mathrm{X}$ axis, $\mathrm{Y}$ axis and $\mathrm{Z}$ axis respectively.

The mechanical model of the proposed five-axis machine tool considered in this paper as shown in Fig.1, is the basic option of the planned reconfigurable multi-axis machining system for HSCmilling of aluminum, epoxy tooling board as well as small size model making, plastic and foam machining. The machine has a two-DOF structure of parallel type to execute one translational 
motion and rotational motion on working table. Compare with the general commercial machine tools, the proposed machine is similar to the type of Rotary Table and Pivoting Spindle Head.

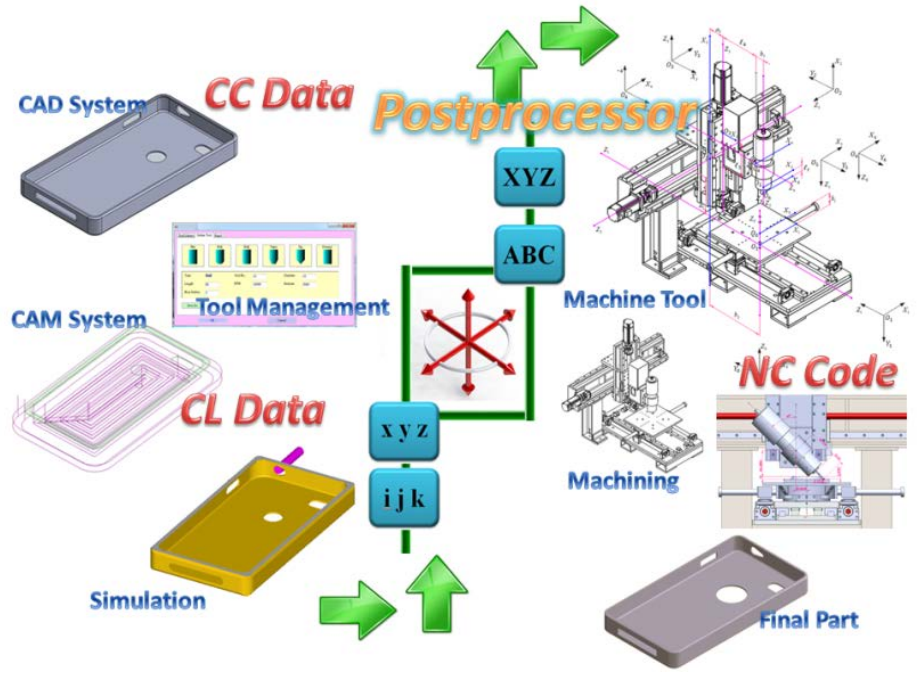

Fig. 1 A flow chart of NC code creation process.

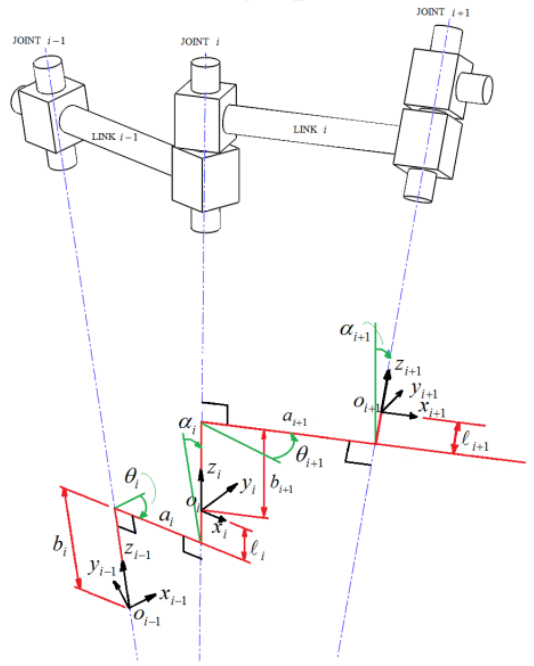

Fig. 2 Modified D-H for spatial linkages.

Modified D-H Representation for Forward Kinematics. A modified D-H notation is used in this paper. The added fifth parameter $l_{i}$ can ensure that the joint is located on the link. That means the adjacent two links can be assembled on correct locations by the transformation matrix. The transformation matrix between adjacent coordinate frames for the modified D-H notation changes as follows:

$$
\begin{aligned}
& { }^{i-1} H_{i}=\operatorname{Trans}\left(0,0, b_{i}\right) \operatorname{Rot}\left(z, \theta_{i}\right) \operatorname{Trans}\left(a_{i}, 0,0\right) \operatorname{Rot}\left(x, \alpha_{i}\right) \operatorname{Trans}\left(0,0, \ell_{i}\right) \\
& =\left[\begin{array}{cccc}
\cos \theta_{i} & -\sin \theta_{i} \cos \alpha_{i} & \sin \theta_{i} \sin \alpha_{i} & \ell_{i} \sin \theta_{i} \sin \alpha_{i+} a_{i} \cos \theta_{i} \\
\sin \theta_{i} & \cos \theta_{i} \cos \alpha_{i} & -\cos \theta_{i} \sin \alpha_{i} & -\ell_{i} \cos \theta_{i} \sin \alpha_{i}+a_{i} \sin \theta_{i} \\
0 & \sin \alpha_{i} & \cos \alpha_{i} & \ell_{i} \cos \alpha_{i}+b_{i} \\
0 & 0 & 0 & 1
\end{array}\right]
\end{aligned}
$$

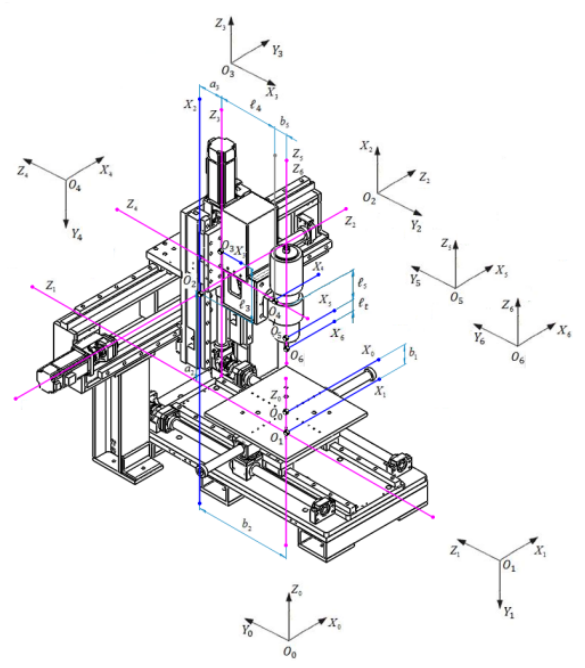

\begin{tabular}{cccccc}
\hline Link/Parameter & $b_{i}$ & $\theta_{i}$ & $a_{i}$ & $\alpha_{i}$ & $\ell_{i}$ \\
\hline $0 / 1$ & $-b_{1}$ & $\theta_{1}$ & 0 & $-\pi / 2$ & 0 \\
$1 / 2$ & $b_{2}$ & $-\pi / 2$ & $a_{2}$ & $-\pi / 2$ & 0 \\
$2 / 3$ & $b_{3}$ & $\pi / 2$ & $a_{3}$ & $\pi / 2$ & $\ell_{3}$ \\
$3 / 4$ & $-b_{4}$ & $\pi / 2$ & 0 & $-\pi / 2$ & $\ell_{4}$ \\
$4 / 5$ & $-b_{5}$ & $\theta_{2}$ & 0 & $\pi / 2$ & $\ell_{5}$ \\
$5 / 6$ & 0 & 0 & 0 & 0 & $\ell_{t}$
\end{tabular}

Fig. 3 5-DOF machine tool.

As shown in Fig. 2, the coordinate systems $O_{i}$ and $O_{i+1}$ are defined as follows. The origin $O_{i}$ is located on $Z_{i}$ of the $i^{\text {th }}$ link and the common normal to both $i^{\text {th }}$ and $(i+1)^{\text {th }}$ joint axis, the $Z_{i}-$ axis 
is coincident with the $i^{\text {th }}$ joint axis, the $X_{i}$-axis is along the common normal to both $i^{\text {th }}$ and $(i+1)^{\text {th }}$ joint axis, and the $Y_{i}$-axis is determined by the cross product of the $Z_{i}$-axis and $X_{i}$-axis .

After the coordinate systems are defined, the D-H parameters can be obtained as:

$b_{i}$ : the distance from point $O_{i-1}$ to the common normal along $Z_{i-1}-$ axis ,

$\theta_{i}$ : the angle between the $X_{i-1}$-axis and the $X_{i}$-axis measured about the $Z_{i-1}$-axis,

$a_{i}$ : the distance between $Z_{i-1}$-axis and $Z_{i}$-axis along the common normal,

$\alpha_{i}$ : the angle between the $Z_{i-1}$-axis and the $Z_{i}$-axis measured about the $X_{i}$-axis,

$l_{i}$ : the distance from the common normal to point $O_{i-1}$ along $Z_{i}$-axis .

The geometric parameters for the example machine tool shown in Fig. 3 are listed in Table 1. Link 0 and link1 implement a rotary variable. Link 1 and link 2, link 2 and link 3, link 3 and link 4 all implement translate variables. Link 4 and link 5 implement another rotary variable. Finally, link 5 and link 6 employ the cutting tool length.

The forward kinematics is to find the relative position and orientation of any two designated members given the geometric structure of the manipulator and the values of a number of joint positions equal to the number of degrees of freedom of the mechanism. For the proposed serialchain machine shown in Fig.3 the transformation is

${ }^{0} H_{6}=\left[\begin{array}{cccc}\operatorname{Cos} \theta_{1} \operatorname{Cos} \theta_{2} & -\operatorname{Sin} \theta_{1} & \operatorname{Cos} \theta_{1} \operatorname{Sin} \theta_{2} & -\ell_{t} \operatorname{Cos} \theta_{1} \operatorname{Sin} \theta_{2}-\ell_{5} \operatorname{Cos} \theta_{1} \operatorname{Sin} \theta_{2}+b_{5} \operatorname{Sin} \theta_{1}+\ell_{4} \operatorname{Sin} \theta_{1}+a_{3} \operatorname{Sin} \theta_{1}+b_{3} \operatorname{Cos} \theta_{1}-b_{2} \operatorname{Sin} \theta_{1} \\ \operatorname{Sin} \theta_{1} \operatorname{Cos} \theta_{2} & \operatorname{Cos} \theta_{1} & \operatorname{Sin} \theta_{1} \operatorname{Sin} \theta_{2} & -\ell_{y} \operatorname{Sin} \theta_{1} \operatorname{Sin} \theta_{2}-\ell_{5} \operatorname{Sin} \theta_{1} \operatorname{Sin} \theta_{2}-b_{5} \operatorname{Cos} \theta_{1}-\ell_{4} \operatorname{Cos} \theta_{1}-a_{3} \operatorname{Cos} \theta_{1}+b_{3} \operatorname{Sin} \theta_{1}+b_{2} \operatorname{Cos} \theta_{1} \\ -\operatorname{Sin} \theta_{2} & 0 & \operatorname{Cos}_{2} & -\ell_{t} \operatorname{Cos} \theta_{2}-\ell_{5} \operatorname{Cos} \theta_{2}-b_{4}+\ell_{3}+a_{2}-b_{1} \\ 0 & 0 & 0 & 1\end{array}\right](2)$

Using homogeneous transformations can provide a compact notation, but are computationally inefficient for solving the forward kinematics problem. A reduction in computation can be achieved by separating the position and orientation portions of the transformation

${ }^{0} H_{6}=\left[\begin{array}{cccc}{ }^{0} I_{6} & { }^{0} J_{6} & { }^{0} K_{6} & { }^{0} P_{6} \\ 0 & 0 & 0 & 1\end{array}\right]$

where

${ }^{0} P_{6}=\left[\begin{array}{c}-\ell_{t} \operatorname{Cos} \theta_{1} \operatorname{Sin} \theta_{2}-\ell_{5} \operatorname{Cos} \theta_{1} \operatorname{Sin} \theta_{2}+b_{5} \operatorname{Sin} \theta_{1}+\ell_{4} \operatorname{Sin} \theta_{1}+a_{3} \operatorname{Sin} \theta_{1}+b_{3} \operatorname{Cos} \theta_{1}-b_{2} \operatorname{Sin} \theta_{1} \\ -\ell_{y} \operatorname{Sin} \theta_{1} \operatorname{Sin} \theta_{2}-\ell_{5} \operatorname{Sin} \theta_{1} \operatorname{Sin} \theta_{2}-b_{5} \operatorname{Cos} \theta_{1}-\ell_{4} \operatorname{Cos} \theta_{1}-a_{3} \operatorname{Cos} \theta_{1}+b_{3} \operatorname{Sin} \theta_{1}+b_{2} \operatorname{Cos} \theta_{1} \\ -\ell_{t} \operatorname{Cos} \theta_{2}-\ell_{5} \operatorname{Cos} \theta_{2}-b_{4}+\ell_{3}+a_{2}-b_{1}\end{array}\right]=\left[\begin{array}{c}P_{x} \\ P_{y} \\ P_{z}\end{array}\right] \quad{ }^{0} K_{6}=\left[\begin{array}{c}\operatorname{Cos} \theta_{1} \operatorname{Sin} \theta_{2} \\ \operatorname{Sin} \theta_{1} \operatorname{Sin} \theta_{2} \\ \operatorname{Cos} \theta_{2}\end{array}\right]=\left[\begin{array}{c}K_{x} \\ K_{y} \\ K_{z}\end{array}\right]$

Inverse Kinematic Model With Virtual C-Axis. For direct kinematic modeling, given the activejoint variables, it is straightforward to uniquely define the position and orientation of the tool tip. The inverse kinematics problem for a serial-chain manipulator is to find the values of the joint positions given the position and orientation of the end-effector relative to the base and the values of all of the geometric link parameters. Once again, this is a simplified statement applying only to serial chains. A more general statement is: given the relative positions and orientations of two members of a mechanism, find the values of all of the joint positions. This amounts to finding all of the joint positions given the homogeneous transformation between the two members of interest. Reviewing the formulation of this transformation of Eqs. (2)-(4), it is clear that the inverse kinematics equations can be found as follows

$$
\begin{aligned}
& b_{3}=\left(P_{y}\right) \operatorname{Sin} \theta_{1}+\left(P_{x}\right) \operatorname{Cos} \theta_{1}+\left(\ell_{t}+\ell_{5}\right) \operatorname{Sin} \theta_{2} \\
& b_{2}=\left(-P_{x}\right) \operatorname{Sin} \theta_{1}+\left(P_{y}\right) \operatorname{Cos} \theta_{1}+a_{3}+b_{5}+\ell_{4} \\
& b_{4}=-\ell_{t} \operatorname{Cos} \theta_{2}-\ell_{5} \operatorname{Cos} \theta_{2}-b_{1}+a_{2}+\ell_{3}-P_{z} \\
& \theta_{1}=\operatorname{Tan}^{-1}\left(\frac{K_{y}}{K_{x}}\right) \\
& \theta_{2}=\operatorname{Cos}^{-1}\left(K_{z}\right)
\end{aligned}
$$


From Table 1, the solution to the inverse position kinematics problem for such a structure is summarized as: parameter $b_{2}$ represents the freedom of $\mathrm{X}$-axis, $b_{3}$ represents the freedom of $\mathrm{Y}$ axis, $b_{4}$ represents the freedom of Z-axis, $\theta_{2}$ represents the freedom of B-axis, and $\theta_{1}$ represents the freedom of C-axis, respectively. From the proposed 5-axis mechanism, if $\mathbf{p}=\left[\begin{array}{llllll}P_{x} & P_{y} & P_{z} & K_{x} & K_{y} & K_{z}\end{array}\right]^{T}$ as a CL position vector in the work piece space, and $\mathbf{q}=\left[\begin{array}{lllll}b_{3} & b_{2} & b_{4} & \theta_{1} & \theta_{2}\end{array}\right]^{T}$ as a joint variable vector in the joint space. As described above, the forward kinematics equation can be derived as $\mathbf{p}=\mathbf{f}(\mathbf{q})$, the differential relationships of the kinematics is written as $\delta \mathbf{p}=\mathbf{J}(\mathbf{q}) \delta \mathbf{q}$, where $\mathbf{J}(\mathbf{q})=\partial \mathbf{f} / \partial \mathbf{q}$ is the Jacobian matrix. Based on Eq. (4), the Jacobian matrix can be calculated.

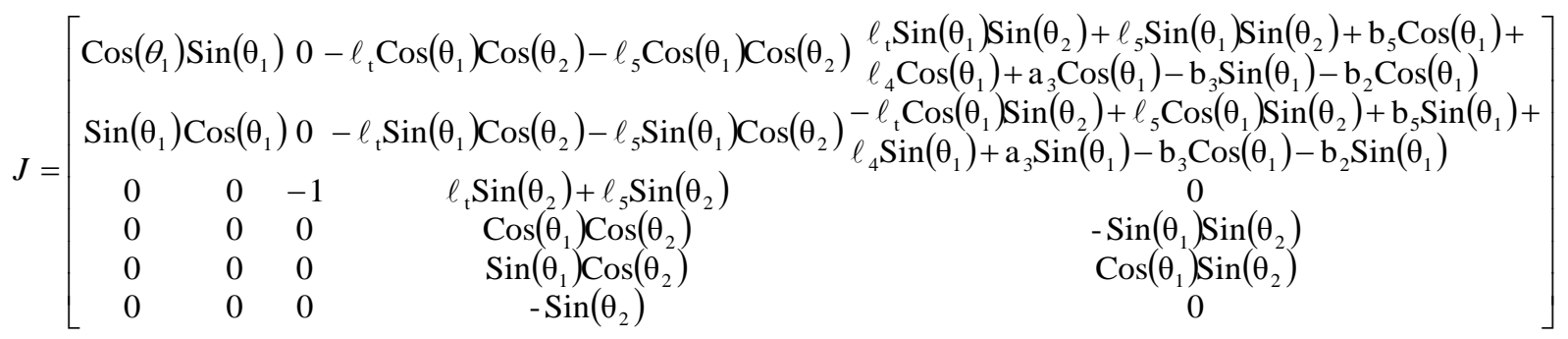

From Eq. (4), since $\left[K_{x} K_{y} K_{z}\right]$ are the direction cosines of the tool axis vector, $K_{x}^{2}+K_{y}^{2}+K_{z}^{2}=1$. Substitute the geometric dimensions of the proposed machine into Eq. (10), the determinant of Jacobian matrix is calculated as

$\operatorname{Det} \mathbf{J}(\mathbf{q})=\partial \mathbf{f} / \partial \mathbf{q}=-\operatorname{Cos}\left(\theta_{1}\right)^{4} \operatorname{Cos}\left(\theta_{2}\right) \operatorname{Sin}\left(\theta_{2}\right)-2 \operatorname{Cos}\left(\theta_{1}\right)^{2} \operatorname{Sin}\left(\theta_{2}\right)^{2} \operatorname{Sin}\left(\theta_{2}\right) \operatorname{Cos}\left(\theta_{2}\right)-\operatorname{Sin}\left(\theta_{1}\right)^{4} \operatorname{Sin}\left(\theta_{2}\right) \operatorname{Cos}\left(\theta_{2}\right)(10)$ The solution of $\operatorname{Det} \mathbf{J}(\mathbf{q})=0$ is $\theta_{2}=k \pi / 2$, where $k$ is integer. Since $\pi / 3>\theta_{2}>-\pi / 3$, the singular configuration is found at $\theta_{2}=0$.

\section{Conclusions}

This paper has proposed a methodology based on the generalized kinematics model for a hybrid parallel-serial five-axis postprocessor. The analytical equations of NC data for various five-axis machine tools with three orthogonal linear axes and two orthogonal rotational axes can be explicitly expressed in terms of CL data. Moreover, the NC data for the specific virtual $C$-axis machine tool can be transformed to the hybrid parallel-serial five-axis machine tool configuration using the derived analytical equations so that the portability of the NC data can be greatly promoted. The presented methodology shows a general procedure that can make the generation of hybrid parallelserial five-axis postprocessors more systematically and efficiently.

\section{Acknowledgment}

The authors gratefully acknowledge the supports of the National Science Council in Taiwan through project number NSC 101-2221-E-212-018-.

\section{Reference}

[1] L. Radavelli, R. Simoni, E. De Pieri and D. Martins. Vol.XXXI (2012), p. 2833-2848.

[2] S Shoham, M. and Ben-Horin, P. Vol. 28 (2009), p. 127-141.

[3] E. Sariyildiz, E. Cakiray and H. Temeltas. Vol. 8 (2011), p. 9-24.

[4] E. Sariyildiz and H. Temeltas. IEEE (2009), p. 338-343.

[5] C.H. She and R.S. Lee. Vol. 2 (2000), p.131-141. 